\title{
Theoretical analysis of capillary performance of triangular microgrooves
}

\author{
T.-S. Sheu, J.-S. Kuo, P.-P. Ding, P.-H. Chen
}

\begin{abstract}
The purpose of this study is to analyze the capillary performance of triangular microgrooves. The influences of several major of parameters on the capillary performance (wetted axial length) of triangular microgrooves are discussed theoretically. One dimensional, nonlinear and contact angle possessed differential and algebraic equations are both used for the theoretical analyses of triangular microgrooves. In this study, the curvature radius, cross section area, and distribution of pressure and velocity of working fluid in microgrooves are considered. Besides, the mutual effects among inertial force, body force, capillary force, and friction force are also discussed. The significance of contact angle and hydraulic diameter to the prediction of capillary performance for microgrooves are both demonstrated by the proposed algebraic solutions.

$h_{f g}$

$P_{\bar{P}}$

$\bar{P}$

$q^{\prime \prime}$

$r$

$\bar{R}$

$\operatorname{Re}$

$S_{w l}$

$v$

$v_{l}$

$\bar{V}_{l}$

$w$

$x$

$\bar{X}$
\end{abstract}

Keywords Capillary, Microgrooves, Contact angle, Micro heat pipe

\section{List of symbols}

$A_{l} \quad$ cross-section area of working fluid in microgroove

$\overline{A_{l}} \quad$ dimensionless cross-section area of working fluid in microgroove

$\mathrm{B}_{0} \quad$ Bond Number

$C_{1} \quad$ constant of Eq. (20)

$C_{2} \quad$ constant of Eq. (21)

$C_{3} \quad$ constant of Eq. (22)

$d_{h, l} \quad$ hydraulic diameter of liquid

$E_{1} \quad$ dimensionless inertial force of Eq. (38)

$E_{2} \quad$ dimensionless body force of Eq. (39)

$E_{3}$ dimensionless friction force of solid-liquid interface of Eq. (40)

$f \quad$ Fanning friction factor

$F_{l, p} \quad$ surface pressure of liquid

$F_{w l . f}$ friction force of solid-liquid interface

$g$ gravity acceleration

$h(x)$ the distance of the center of curvature radius of working fluid to the bottom of microgroove

Received: 22 January 2003 / Accepted: 11 June 2003

T.-S. Sheu

Department of Mechanical Engineering,

Chinese Military Academy, Kaoshiung, Taiwan 830

J.-S. Kuo, P.-P. Ding, P.-H. Chen ( $\varangle)$

Department of Mechanical Engineering,

National Taiwan University, Taipei, Taiwan 10673

e-mail:phchen@ntu.edu.tw
Greek symbols

$\alpha$

$\beta$

$\varepsilon_{1}$

$\varepsilon_{2}$

$\varepsilon_{3}$

$\mu$

$\theta$

$\rho$

$\sigma$

$\tau$

$v$

pressure

heat flux

velocity

velocity latent heat of working fluid

dimensionless pressure

curvature radius

dimensionless curvature radius

Reynolds Number

wetted side length

dimensionless velocity

half width of the top of microgroove

Cartesian coordinate

dimensionless Cartesian coordinate

\author{
half angle of the bottom of microgroove \\ inclined angle \\ constant of Eq. (25) \\ constant of Eq. (26) \\ constant of Eq. (27) \\ viscosity \\ contact angle \\ density \\ surface tension \\ shear stress \\ kinetic viscosity
}

Subscript

$0 \quad$ quantities at $x=0$

1

\section{Introduction}

Due to the high thermal efficiency of liquid thin film on solid surface with microgrooves, many heat transfer devices, such as heat pipe, evaporator, heat sink, and thermal control components of electronic devices, employ this distinctive physical character as the operation principle.

Particularly, the heat pipes that use microgrooves as capillary wicks can effectively increase the heat exchange rates on both evaporation and condensation ends. The capillary force of liquid thin film in microgrooves is the driving force that induces the working fluid from the condensation end to the evaporation end. During operation, if the heat flux that is applied to the heat pipe exceeds the heat capacity of capillary wicks, the evaporation end will dry out and hence result in damage. For this reason, understanding the heat flow behavior of the working fluid in 
capillary wicks is included as the design criterion of heat pipes. From the mechanical viewpoint, the research into the heat flow information of the working fluid on the surface of microgrooves can effectively improve the performance of heat pipes.

Holm and Goplen [1] developed a theoretical model for describing the heat and mass transfer from a wetted groove surface. As shown in Fig. 1, they divided the extended meniscus formed on the heated wall into three regions, and developed theoretical analysis for every region. The first is the so-called "Equilibrium Thin Film Region", formed at the upper part of the extended meniscus. In this region, the short-range adhesion force prevents evaporation and there is no heat dissipation from this region. The second is called "Evaporating Thin Film Region", formed just below the equilibrium thin film. In this region, the primary effects are short-range adhesion force and capillary force. With increasing thin film thickness, the short-range adhesion force decreases, but capillary force increases. The third is called "Intrinsic Meniscus", formed at the bottom of the extended meniscus. In this region, the capillary force is the dominant factor. The thin film thickness is large enough to introduce significant increases in thermal resistance due to conduction. $\mathrm{Xu}$ and Carey [2] proposed an analytical model that can be used to predict the heat transfer characteristics of film evaporation on a V-shaped microgroove. Their predicted results agree fairly well with experimental data. Stephan and Busse [3] presented an analytical model for the radial heat transfer of a grooved heat pipe evaporator. The temperature distributions in the solid area of microgrooves were provided in their study. Stroes et al. [4] conducted an experimental study to investigate the effects of heat flux and the inclination angle on the capillary performance of both rectangular and triangular channels. For comparison with experimental data of Stroes et al. [4], $\mathrm{Ha}$ and Peterson [5] presented an analytical investigation to predict the axial dryout location in V-shaped

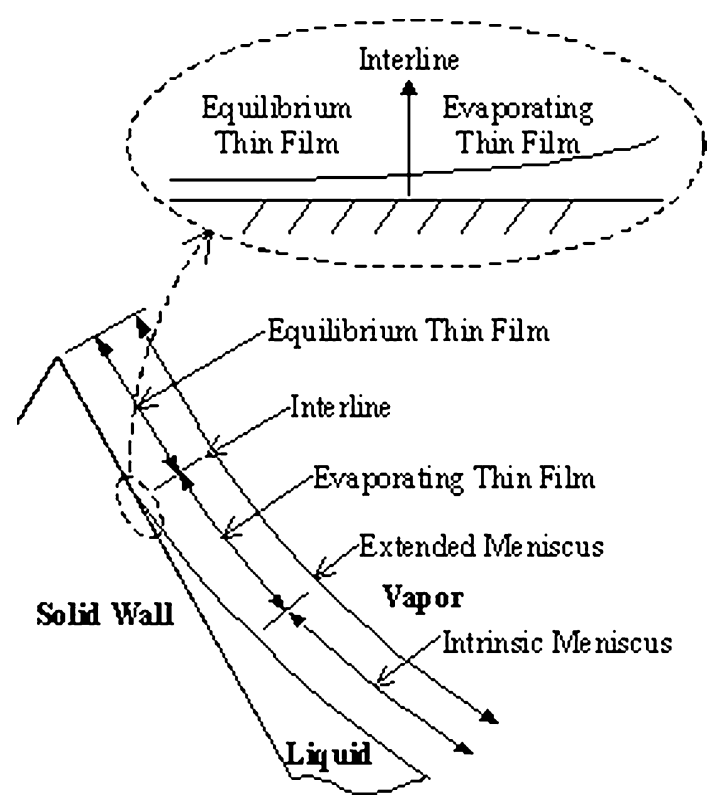

Fig. 1. Thin film definition formed by the interface of working fluid and groove surface microgrooves. They considered the effects of heat flux, the material properties of the working fluid and the geometry of the grooves on the axial dryout location in V-shaped microgrooves. Khrustalev and Faghri [6] developed a detailed mathematical model that described the influence of the geometry on the capillary force in grooves. The model also accounted for the effects of interfacial thermal resistance, disjoining pressure, and surface roughness for a given meniscus contact angle. Ha and Peterson [7] established a V-shaped mathematical model including the effect of the angle of inclination to describe the axial flow of an evaporating thin film through a $\mathrm{V}$-shaped microgroove. Peterson and $\mathrm{Ha}[8]$ used above model, and simplified the original complex nonlinear differential governing equation as an approximate algebraic model. They also used the modeling results to predict the axial capillary performance, and compared them to the results of the experimental investigation. Sheu et al. [9] studied the effect of surface characteristics on the capillary performance of a triangular microgrooves plate with a chemically etched surface texture containing micro cavities.

Since the effects of dominant forces, contact angle and hydraulic diameter on capillary performance of microgroove have not been demonstrated in most literature studies $[5,8]$, the present study offers theoretical analysis to discuss the effects of various factors on the triangular microgroove capillary performance (wetted axial length). The factors considered in this investigation include heat flux, the angle of inclination, hydraulic diameter and contact angle. Moreover, a one-dimensional, nonlinear differential equation and an algebraic equation including the effect of contact angle are developed. Based on this theoretical analysis, the meniscus curvature radius of working fluid, cross-section area, pressure and velocity distribution are discussed, and the mutual effects of inertial force, body force, capillary force, and friction force are also discussed.

\section{2}

\section{Theoretical analyses model}

Figure 2 shows the physical model. The principle purpose of this model is to simulate the operation mode of groovetyped heat pipes, and is divided into three regions including evaporator section, adiabatic section and condenser section. In this model, uniform heat flux will be applied on the microgrooves plate in an evaporating section. The condenser section is immersed in working fluid. The section between evaporator and condenser is an adiabatic section that has no heat flux passing through. The intersectional point of adiabatic and evaporator section is defined as the junction point (or the origin point, $x=0$ ). Due to the capillary effect, the working fluid overcomes gravity and friction and ascends to the top of the microgrooves. The maximum point of ascension is called the dryout point. Besides, $\beta$ is the angle between test sample and horizontal. The geometry of triangular microgrooves is shown in Fig. 3.

The flow of working fluid in microgrooves mainly relies on capillary force. Therefore, it is assumed in the present study that with a laminar flow, steady state, and constant 


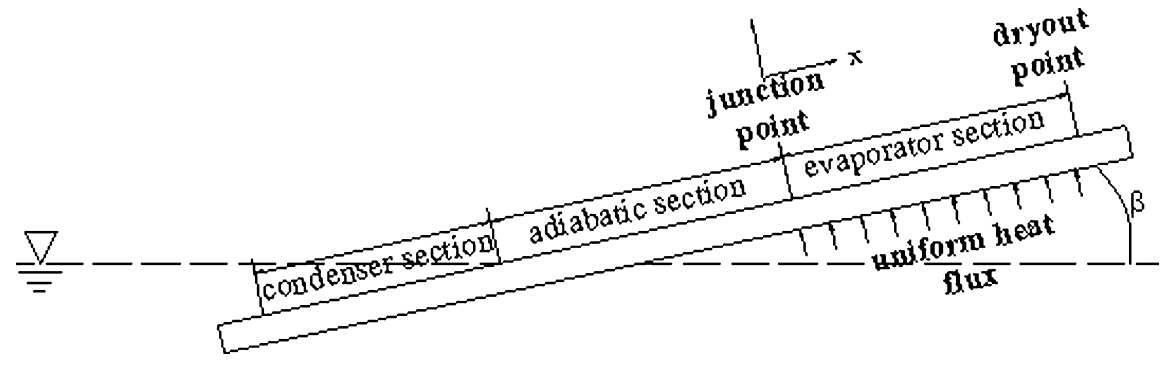

Fig. 2. Physical model

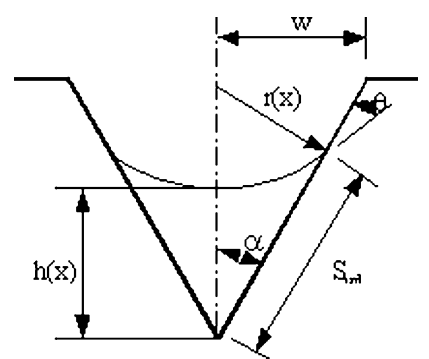

Fig. 3. The geometry shape of a triangular microgroove

properties Newtonian fluid, the vapor pressure is constant, and the flow behavior of working fluid happens only in microgrooves. In other words, the working fluid does not flood over the top of the microgrooves. For very small grooves, the radius curvature of meniscus formed by working fluid on the cross section can be considered as a constant. When microgrooves are heated, the intrinsic meniscus contracts towards groove bottoms. This contraction causes the reduction of radius curvature, and the radius decreases along $x$-axis down to a minimum. On the cross section of microgrooves, the working fluid above the intrinsic meniscus continues to extend toward the apex of the grooves due to the dispersion force, and forms a liquid thin film. Above the intrinsic meniscus region, the behavior of liquid thin film is dominated by dispersion force, and the intrinsic meniscus is limited by the axial flow primarily. The axial heat transfer is much less than the normal heat transfer of the microgrooves plate, so the axial heat transfer can be ignored.

From the control volume of liquid working fluid in the microgrooves, the momentum balance equation is given as

$\int_{V} \rho_{l} g_{x} \mathrm{~d} V+\int_{s}(T \cdot \mathrm{d} s)_{x}=\int_{s} \rho_{l} v_{l}\left(\hat{v}_{l} \cdot \hat{n}\right) \mathrm{d} s$

where $\rho_{l}$ is the density of liquid, $g$ is acceleration of gravity, $T$ is the stress tensor, and $s$ is the boundary of the control volume of the surface area.

The first term of left side in Eq. (1) is the gravity term, the second term is the surface tension term, and right side represents the dynamic term. The surface tension includes the surface pressure of liquid $\left(F_{l, p}\right)$, the friction force of solid-liquid interface $\left(F_{w l, f}\right)$ and the friction force of liquid-vapor interface $\left(F_{l v, f}\right)$. Without any interaction on liquid-vapor interface, the atmospheric pressure surrounds the open microgrooves, and no adverse vapor flow on liquid curve surface are assumed. So the friction force of liquid-vapor interface is ignored in this study, without which the surface tension term can be simplified as
$\int_{s}(T \cdot \mathrm{d} s)_{x}=F_{l, p}-F_{w l, f}$

From the force equilibrium of the control volume, the surface pressure is shown as

$F_{l, p}=-\left.p_{l} A_{l}\right|_{x+d x}-p_{l} \mathrm{~d} A_{v}+\left.p_{l} A_{l}\right|_{x}=-A_{l} \mathrm{~d} p_{l}$.

The pressure difference of the liquid-vapor interface can be described by Young-Laplace equation:

$p_{v}-p_{l}=\sigma\left(\frac{1}{r_{1}}+\frac{1}{r_{2}}\right) \approx \frac{\sigma}{r}$.

At each point on the liquid-vapor interface, the surface has two principal radii of curvature, $r_{1}$ and $r_{2}$, in orthogonal planes normal to the surface. And the axial radius $r_{2}$, approaches infinity when compared to another comparatively small radius, $r_{1}$, of the intrinsic meniscus. Thus the surface pressure of the liquid can be expressed as

$F_{l, p}=-A_{l} d\left(p_{v}-\frac{\sigma}{r}\right)$.

Assuming that the vapor pressure and surface tension are constant, Eq. (5) can be simplified as

$F_{l, p}=A_{l} \sigma d\left(\frac{1}{r}\right)$.

The hydraulic diameter and flow velocity of the liquid are expressed respectively as

$d_{h, l}=\frac{4 A_{l}}{S_{w l}}$,

$v_{l}=\frac{\dot{m}_{l}}{\rho_{l} A_{l}}$

where $\dot{m}_{1}$ is the mass flow rate of the liquid, and the Reynolds number is

$\operatorname{Re}_{l}=\frac{\rho_{l} v_{l} d_{h, l}}{\mu_{l}}$.

The shear stress of the solid-liquid interface is

$\tau_{w l}=\frac{1}{2} \rho_{l} v_{l}^{2} f$

where $f\left(=k / \mathrm{Re}_{l}\right)$ is the Fanning friction factor, $k$ is the friction coefficient and has been determined by Ayya-

swamy et al. [10]. The value of $k$ is a quarter of the value given in the work of Ayyaswamy et al. [10], and depends on the contact angle. The friction force of the solid-liquid interface becomes 
$F_{w l, f}=\tau_{w l} S_{w l} \mathrm{~d} x=\frac{2 k \dot{m}_{l} \mu_{l}}{\rho_{l} d_{h, l}^{2}} \mathrm{~d} x$

Substituting Eqs. (6) and (11) into Eq. (2)

$$
\int_{s}(T \cdot \mathrm{d} s)_{x}=A_{l} \sigma d\left(\frac{1}{r}\right)-\frac{2 k \dot{m}_{l} \mu_{l}}{\rho_{l} d_{h, l}^{2}} \mathrm{~d} x \text {. }
$$

In Eq. (1), the right size of the equal sign is the momentum term along the $x$ direction, and from the momentum equilibrium of fluid dynamics Eq. (13) is obtained:

$\int_{s} \rho_{l} v_{l}\left(\bar{v}_{l} \cdot \bar{n}\right) \mathrm{d} S=\dot{m}_{l} \mathrm{~d} v_{l}=\mathrm{d}\left(\dot{m}_{l} v_{l}\right)-v \mathrm{~d} \dot{m}_{l}$.

From energy conservation, the mass flow can be obtained as

$$
\begin{aligned}
\dot{m}_{l} & =-\int_{\dot{m}}^{0} \mathrm{~d} \dot{m}=\frac{1}{h_{f g}} \int_{x_{\max }}^{x} q^{\prime \prime} 2 w(\mathrm{~d} x) \\
& =\frac{2}{h_{f g}} q^{\prime \prime} w\left(x_{\max }-x\right) .
\end{aligned}
$$

Substituting Eq. (14) into Eq. (13), the momentum term becomes

$$
\begin{aligned}
\int_{s} \rho_{l} v_{l}\left(\bar{v}_{l} \cdot \bar{n}\right) \mathrm{d} s= & \frac{4 q^{\prime 2} w^{2}}{h_{f g}^{2} \rho_{l}} \mathrm{~d}\left[\frac{\left(x_{\max }-x\right)^{2}}{A_{l}}\right] \\
& +\frac{4 q^{\prime 2} w^{2}}{h_{f g}^{2} \rho A_{l}}\left(x_{\max }-x\right) \mathrm{d} x .
\end{aligned}
$$

The first term of Eq. (1) is the gravity term, and the gravitational force along $x$ direction is

$$
\int_{v} \rho_{l} g_{x} \mathrm{~d} v=\rho_{l} g A_{l} \sin \beta \mathrm{d} x
$$

Other related geometric parameters, including the cross-section area of fluid, hydraulic diameter and the wetted axial circumference of solid-liquid interface, are expressed as

$$
\begin{aligned}
& A_{l}=C_{1} r^{2}, \\
& d_{h, l}=C_{3} r \\
& S_{w l}=C_{2} r
\end{aligned}
$$

where

$$
\begin{aligned}
C_{1}= & \sin ^{2}(\pi / 2-\alpha-\theta) \tan (\pi / 2-\alpha)+\sin (\pi / 2-\alpha-\theta) \\
& \times \cos (\pi / 2-\alpha-\theta)-(\pi / 2-\alpha-\theta) \\
C_{2}= & \frac{\sin (\pi / 2-\alpha-\theta)}{\sin \alpha} \\
C_{3}= & \frac{4 C_{1}}{C_{2}}
\end{aligned}
$$

Substituting Eqs. (12), (15) and (16) into Eq. (1), leads to

$$
\begin{gathered}
{\left[\frac{8 q^{\prime 2} w^{2}}{C_{1}^{2} h_{f g}^{2} \rho_{l} \sigma} \frac{\left(x_{\max }-x\right)^{2}}{r^{3}}-1\right] \frac{\mathrm{d} r}{\mathrm{~d} x}=-\frac{\rho_{l} g \sin \beta}{\sigma} r^{2}} \\
+\left[\frac{4 k \mu_{l} q^{\prime \prime} w}{C_{1} C_{3}^{2} h_{f g} \rho_{l} \sigma}-\frac{4 q^{\prime \prime 2} w^{2}}{C_{1}^{2} h_{f g}^{2} \rho_{l} \sigma}\right] \frac{\left(x_{\max }-x\right)}{r^{2}} .
\end{gathered}
$$

Ordering Eq. (23) results in

$$
\left[2 \varepsilon_{1} \frac{\left(x_{\max }-x\right)^{2}}{r^{3}}-1\right] \frac{\mathrm{d} r}{\mathrm{~d} x}=-\varepsilon_{2} r^{2}+\left(\varepsilon_{3}-\varepsilon_{1}\right) \frac{\left(x_{\max }-x\right)}{r^{2}}
$$

where $\varepsilon_{1}$ is inertial force defined as

$\varepsilon_{1}=\frac{4 q^{\prime \prime 2} w^{2}}{C_{1}^{2} h_{f g}^{2} \rho_{l} \sigma}$

$\varepsilon_{2}$ is body force defined as

$\varepsilon_{2}=-\frac{\rho_{l} g \sin \beta}{\sigma}$

$\varepsilon_{3}$ is solid-liquid interfacial friction force defined as

$\varepsilon_{3}=\frac{4 k \mu_{l} q^{\prime \prime} w}{C_{1} C_{3}^{2} h_{f g} \rho_{l} \sigma}$

The liquid film fills the whole microgroove. At the junction point of microgroove and working fluid surface, $x=0$ is assumed, and the boundary conditions can be expressed as

$r_{0}=\frac{w}{\sin (\pi / 2-\alpha-\theta)} \quad$ at $x=0$.

Equation (24) is a first order, non-linear ODE that can be solved numerically. However, it will be a better choice to use an approximation instead of the numerical method in obtaining $x_{\max }$. Thus, the polynomial $h(x)$, which satisfies the three boundary conditions given above, is assumed.

$h=h_{0} \quad$ at $x=0$,

$h \approx 0 \quad$ at $x=x_{\max }$,

$\frac{\mathrm{d} h}{\mathrm{~d} x}=0 \quad$ at $x=x_{\max }$.

The quadratic expressions for the assumed polynomial expression for $h(x)$ with only three boundary conditions Eqs. (29)-(31) are

$$
\begin{aligned}
& h(x)=\frac{h_{0}}{x_{\max }^{2}}\left(x-x_{\max }\right)^{2}=\left[\frac{\sin \left(\frac{\pi}{2}+\theta\right)}{\sin \alpha}-1\right] r(x), \\
& \frac{\mathrm{d} r(x)}{\mathrm{d} x}=\frac{2 r_{0}}{x_{\max }^{2}}\left(x-x_{\max }\right) .
\end{aligned}
$$

Substituting Eq. (33) into Eq. (24), produces

$$
\begin{gathered}
{\left[2 \varepsilon_{1} \frac{\left(x_{\max }-x\right)^{2}}{r^{3}}-1\right]\left[\frac{2 r_{0}}{x_{\max }^{2}}\left(x-x_{\max }\right)\right]} \\
=-\varepsilon_{2} r^{2}+\left(\varepsilon_{3}-\varepsilon_{1}\right) \frac{\left(x_{\max }-x\right)}{r^{2}} .
\end{gathered}
$$


With the boundary condition $r=r_{0}$ at $x=0$, Eq. (34) becomes

$x_{\max }=\frac{\varepsilon_{2} r_{0}^{4} \pm r_{0} \sqrt{\varepsilon_{2}^{2} r_{0}^{6}+8\left(\varepsilon_{3}+3 \varepsilon_{1}\right) r_{0}}}{2\left(\varepsilon_{3}+3 \varepsilon_{1}\right)}$.

If the properties of working fluid, heat flux, and contact angle are known, the wetted axial length can be evaluated by Eq. (35).

Dimensionless radius curvature $(\bar{R})$, dimensionless coordinate along $x$ direction $(\bar{X})$ and dimensionless maximum wetted axial length $\left(\bar{X}_{\max }\right)$ are respectively assumed, and defined as

$\bar{R}=\frac{r}{r_{0}}, \quad \bar{X}=\frac{x}{r_{0}}, \quad \bar{X}_{\max }=\frac{x_{\max }}{r_{0}}$.

By substituting Eq. (36) into Eq. (24), the nonlinear differential equation becomes

$$
\begin{aligned}
& {\left[2 E_{1} \frac{\left(\bar{X}_{\max }-\bar{X}\right)^{2}}{\bar{R}^{3}}-1\right] \frac{\mathrm{d} \bar{R}}{\mathrm{~d} \bar{X}}} \\
& =-E_{2} \bar{R}^{2}+\left[E_{3}-E_{1}\right] \frac{\left(\bar{X}_{\max }-\bar{X}\right)}{\bar{R}^{2}} .
\end{aligned}
$$

where $E_{1}, E_{2}$, and $E_{3}$ are the dimensionless inertia force, the dimensionless body force, and the dimensionless solid-liquid interfacial force respectively, and are expressed as

$E_{1}=\frac{\varepsilon_{1}}{r_{0}}$

$E_{2}=\varepsilon_{2} r_{0}^{2}=\mathrm{B}_{0} \times \sin \beta$,

$E_{3}=\frac{\varepsilon_{3}}{r_{0}}$.

The dimensionless boundary condition is

$\bar{R}_{0}=1 \quad$ at $\bar{X}=0$.

Hence, the dimensionless algebraic equation is expressed as

$\bar{X}_{\max }=\frac{E_{2} \pm \sqrt{E_{2}^{2}+8\left(E_{3}+3 E_{1}\right)}}{2\left(E_{3}+3 E_{1}\right)}$.

\section{3}

\section{Results and discussion}

\section{The verification of numerical solution}

\section{and algebraic solution}

By using the numerical method to solve Eq. (37), the curvature of radius and wetted axial length of the working fluid in the microgroove can be obtained, and the cross section area, velocity and pressure distribution can be solved further. However, the advantage of Eq. (42) is that the wetted axial length can be obtained quickly by the algebraic equation. The predicated values of these two theoretical methods are compared to the experimental data (non-etched surface texture cases) of Sheu et al. [9]. Figure 4 shows the relation between wetted axial length and the angle of inclination at $q^{\prime \prime}=5150 \mathrm{~W} / \mathrm{m}^{2}, \theta=0.1^{\circ}$, and all material properties are given under the same

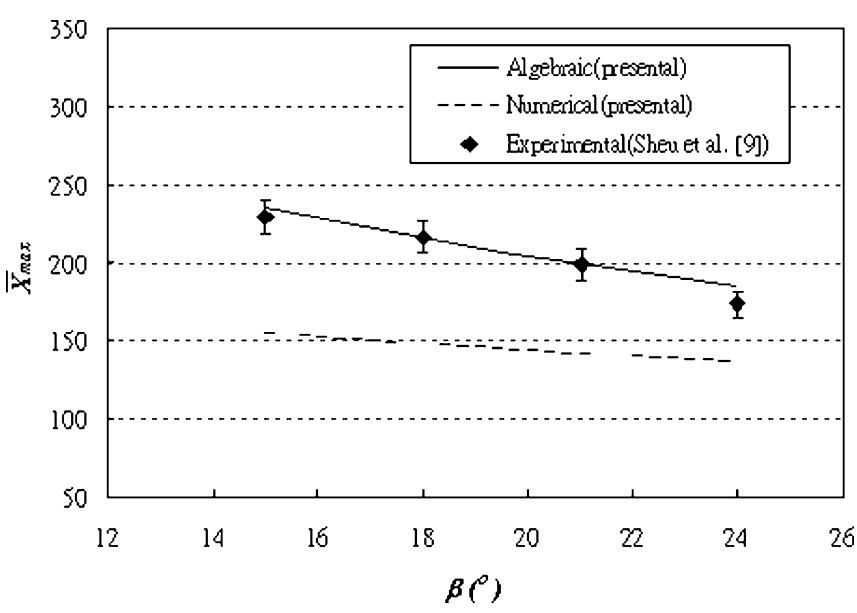

Fig. 4. Wetted axial length of triangular microgroove versus the angle of inclination (methanol, $q^{\prime \prime}=5150 \mathrm{~W} / \mathrm{m}^{2}$ and $\theta=0.1^{\circ}$ )

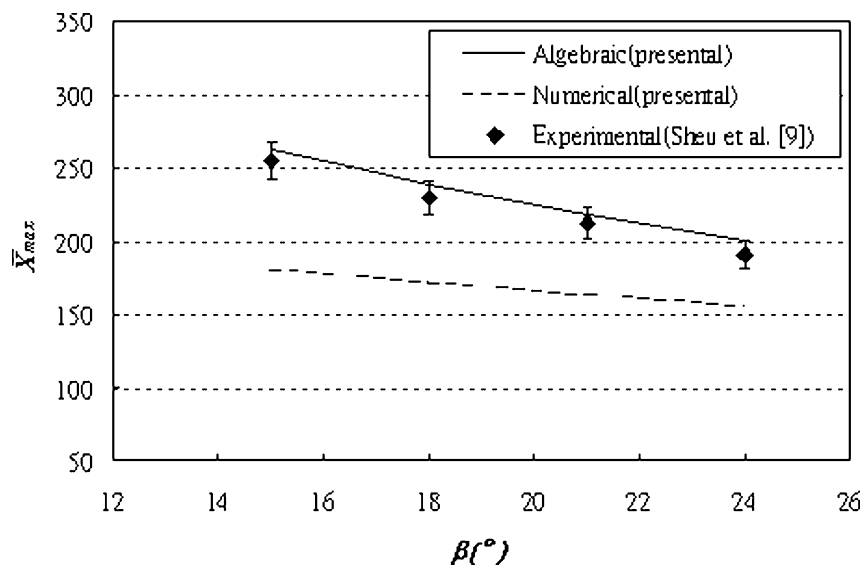

Fig. 5. Wetted axial length of triangular microgroove versus the angle of inclination (methanol, $q^{\prime \prime}=8070 \mathrm{~W} / \mathrm{m}^{2}$ and $\theta=0.1^{\circ}$ )

atmospheric pressure. The results of both methods show larger differences with experimental data at a small inclination angle than at large inclination angle. Although the qualitative trends of the numerical results and the algebraic results are similar, the numerical results have larger differences with experimental results as compared with the algebraic results. The differences decrease with increasing inclination angle. It is obvious that the algebraic data is very close to the experimental data. When heat flux is increased to $8070 \mathrm{~W} / \mathrm{m}^{2}$, the wetted axial length versus the inclination angle is shown in Fig. 5. The trend of this figure is similar to the previous ones, and the algebraic solution is still slightly larger than the experimental data of Sheu et al. [9] (non-etched surface texture).

\section{Mechanism analysis of microgrooves}

In order to clearly understand the mechanism of all influences on the capillary performance in microgroove, the numerical method is used to estimate curvature radius and cross-section area of working fluid, pressure and the distribution of flow velocity. From the variation of these factors in microgroove, the influence on wetted axial length is further discussed. The dimensionless variation of 


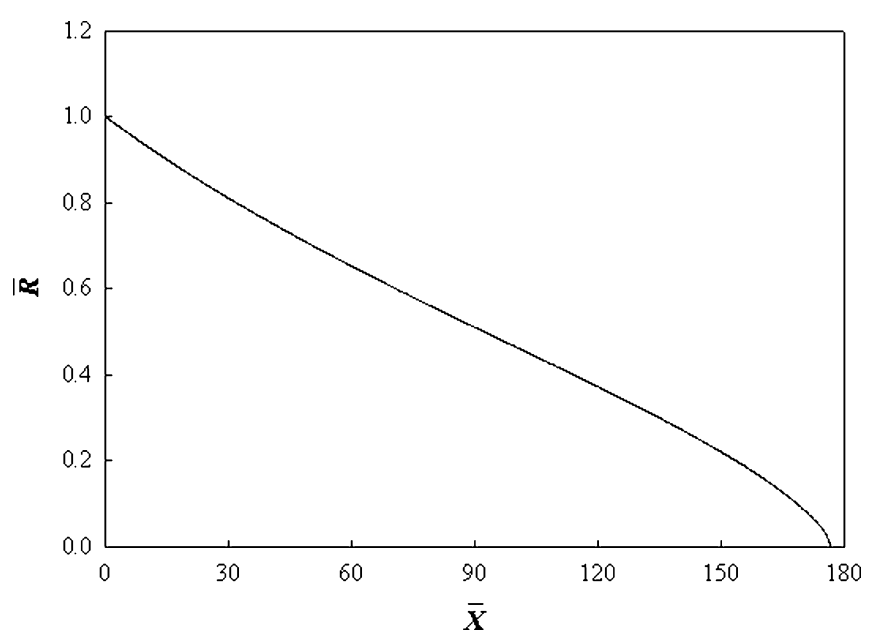

Fig. 6. The variation of curvature radius on liquid surface along $x$ direction (methanol, $q^{\prime \prime}=5150 \mathrm{~W} / \mathrm{m}^{2}, \theta=0.1^{\circ}$ and $\beta=15^{\circ}$ )

the cross-section area, pressure and velocity distribution are expressed as

$\overline{A_{l}}=\frac{A_{l}}{A_{l, 0}}=\frac{\bar{R}^{2}}{\bar{R}_{0}^{2}}$,

$\bar{P}_{l}=\frac{p_{l}}{p_{v}}=1-\frac{\sigma}{p_{v} r}$,

$\bar{V}_{l}=\frac{v_{l}}{v_{l, 0}}=\frac{\bar{X}_{\max }-\bar{X}}{\bar{X}_{\max }} \frac{\bar{R}_{0}^{2}}{\bar{R}^{2}}$.

The example here is to express the variations of curvature of radius and the cross-section area, and the pressure distribution along the axial direction at $q^{\prime \prime}=5150 \mathrm{~W} / \mathrm{m}^{2}$, with methanol as the working fluid and $\theta=0.1^{\circ}$. Figure 6 shows the variation of curvature of radius on working fluid surface along axial direction in the microgrooves. Because of the capillary force and evaporation, the curvature of radius on liquid surface falls along the evaporating section to the dryout point, and there is a rapid drop near dryout point. From Eq. (43), the variation of the cross-section area of the working fluid along the axial direction is obtained and shown in Fig. 7. This result indicates that the cross-section area of the working fluid in the microgrooves falls with a concave form along the axial direction. The reduction of the curvature of radius indicates that the liquid surface contracts towards the top of the grooves, and causes the reduction of working fluid in the crosssection area, especially when both the curvature of radius and cross-section area are very small at the evaporating section. However, in constant heat flux condition, in order to maintain mass conservation after the reduction of the cross-section area of the working fluid the flow rate must increase as shown in Fig. 8. The curvature of radius decreases along axial direction, and the vapor pressure varies slightly. Figure 9 shows that the pressure of the working fluid decreases approaching the evaporating region, and a sharp drop takes place near the dryout point. It implies that the wetted axial length has very small effect to the pressure values near the dryout point.

According to the calculation of Eqs. (37), (43), (44) and (45), the variations of curvature of radius, cross-section

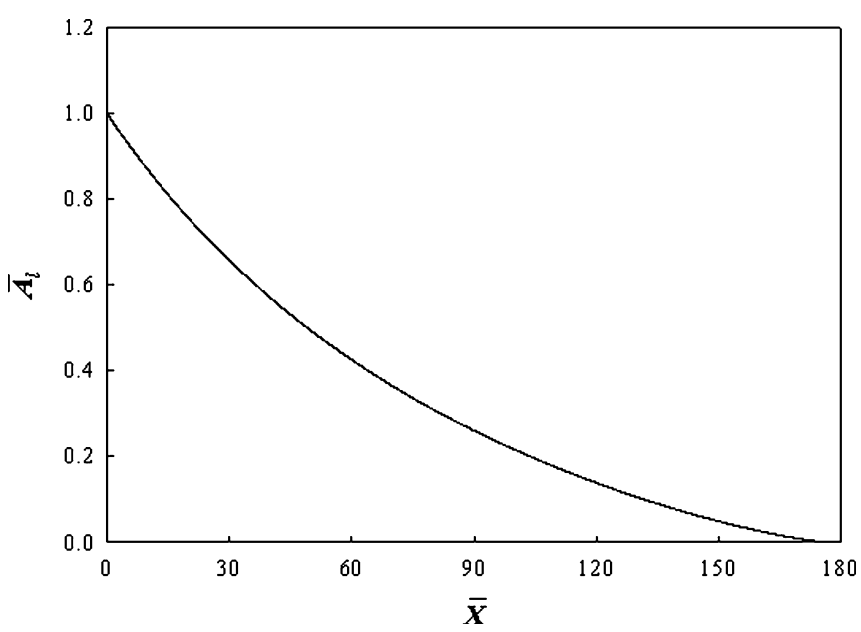

Fig. 7. The variation of the fluidic cross-section area along $x$ direction (methanol, $q^{\prime \prime}=5150 \mathrm{~W} / \mathrm{m}^{2}, \theta=0.1^{\circ}$ and $\beta=15^{\circ}$ )

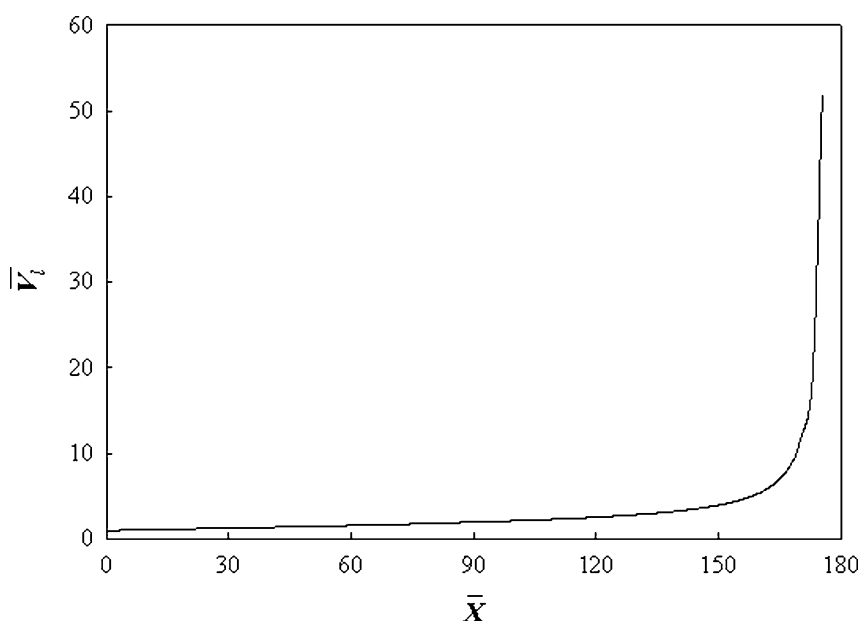

Fig. 8. The distribution of flow velocity along $x$ direction (methanol, $q^{\prime \prime}=5150 \mathrm{~W} / \mathrm{m}^{2}, \theta=0.1^{\circ}$ and $\beta=15^{\circ}$ )

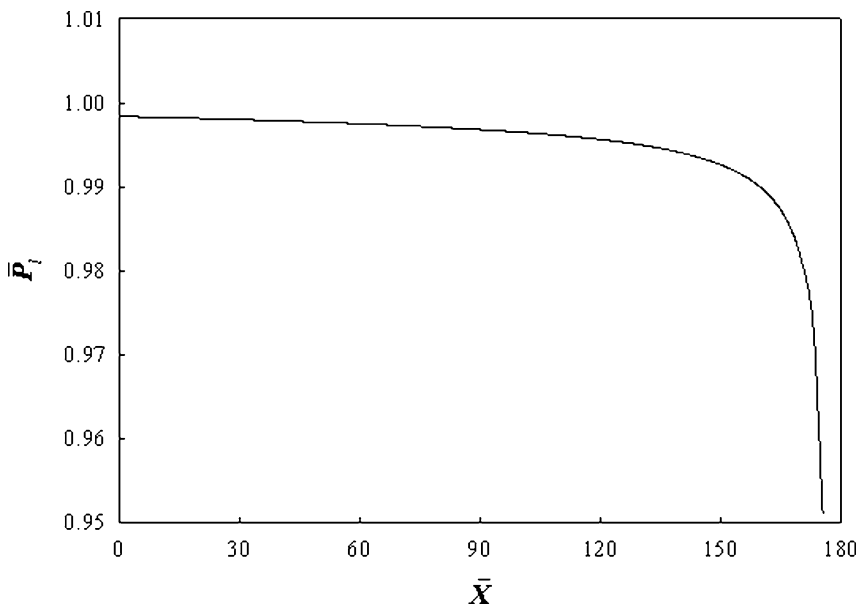

Fig. 9. The pressure distribution along $x$ direction (methanol, $q^{\prime \prime}=5150 \mathrm{~W} / \mathrm{m}^{2}, \theta=0.1^{\circ}$ and $\beta=15^{\circ}$ )

area, flow rate and pressure distribution of working fluid are comprehended. Besides, the influences of all forces on capillary performance in microgrooves are investigated 


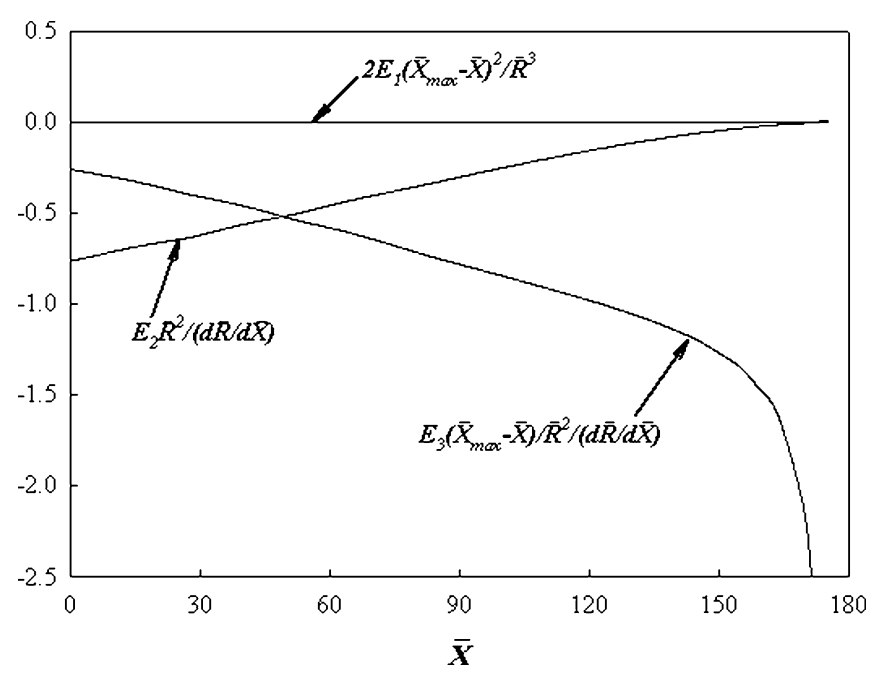

Fig. 10. The variation of every term in Eq. (37) along $x$ direction (methanol, $q^{\prime \prime}=5150 \mathrm{~W} / \mathrm{m}^{2}, \theta=0.1^{\circ}$ and $\beta=15^{\circ}$ )

based on force equilibrium. Figure 10 shows the variations of all terms of Eq. (37) along $\bar{X}$-direction under the operating conditions $q^{\prime \prime}=5150 \mathrm{~W} / \mathrm{m}^{2}, \theta=0.1^{\circ}$, and $\beta=15^{\circ}$. There are different dominating forces at different regions in a microgroove. These forces are capillary force, body force (gravitational force) and friction force of solid-liquid interface. According to the order analysis of Eq. (37), all terms have the same order except $2 E_{1}\left(\bar{X}_{\max }-\bar{X}\right)^{2} / \bar{R}^{3}$ term that is three orders less than all other terms. The $E_{2} \bar{R}^{2} /(\mathrm{d} \bar{R} / \mathrm{d} \bar{X})$ term, which includes $\mathrm{B}_{0}$ number $\left(=\rho_{1} g r^{2} / \sigma\right)$, has a large influence on the body force due to its larger mass and curvature of radius. With the increase of $\bar{X}$ and decrease of the curvature of radius and mass of the working fluid, the influence of the capillary force rises and surpasses the body force gradually. However, the effects of body force and capillary force are equally important at $\bar{X}=0.5 \bar{X}_{\max }$. The $E_{3}\left(\bar{X}_{\max }-\bar{X}\right) / \bar{R}^{2} /$ $(\mathrm{d} \bar{R} / \mathrm{d} \bar{X})$ term shows the friction force effect of solidliquid interface. Since the flow rate of working fluid decreases along $\bar{X}$-direction, the friction force of solidliquid interface is also reduced slightly. On the whole, the dominant forces are the body force and the friction force of the solid-liquid interface at the half front region of the microgroove $\left(\bar{X}<0.5 \bar{X}_{\max }\right)$, and the dominant forces become the capillary force and the friction force of the solidliquid interface at the back region of the microgroove $\left(\bar{X}>0.5 \bar{X}_{\max }\right)$.

The effect of contact angle and hydraulic diameter The contact angle is the included angle between the interface of the working fluid and the solid wall when the working fluid contacts the solid wall. It depends on the surface characteristic and physical properties of both working fluid and solid surface. Generally speaking, if the contact angle is smaller the capillary force increases. Based on the operation principle of the heat pipe, the principal capillary structure offers capillary force. It is expected that the advance of the capillary has absolute assistance in the performance of the heat pipe. Thus, the contact angle is usually a much considered factor in the design of heat

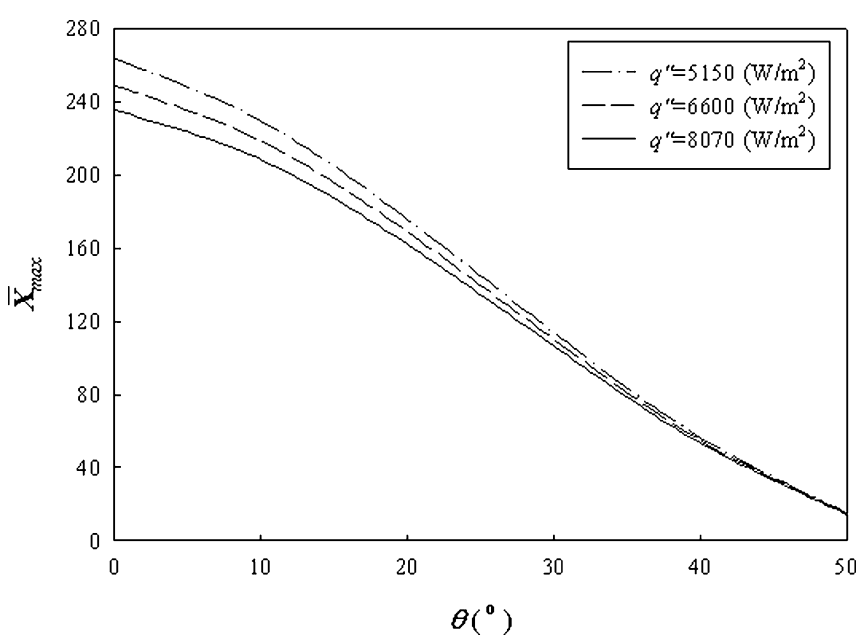

321

Fig. 11. Wetted axial length versus contact angle at different heat fluxes (methanol and $\beta=15^{\circ}$ )

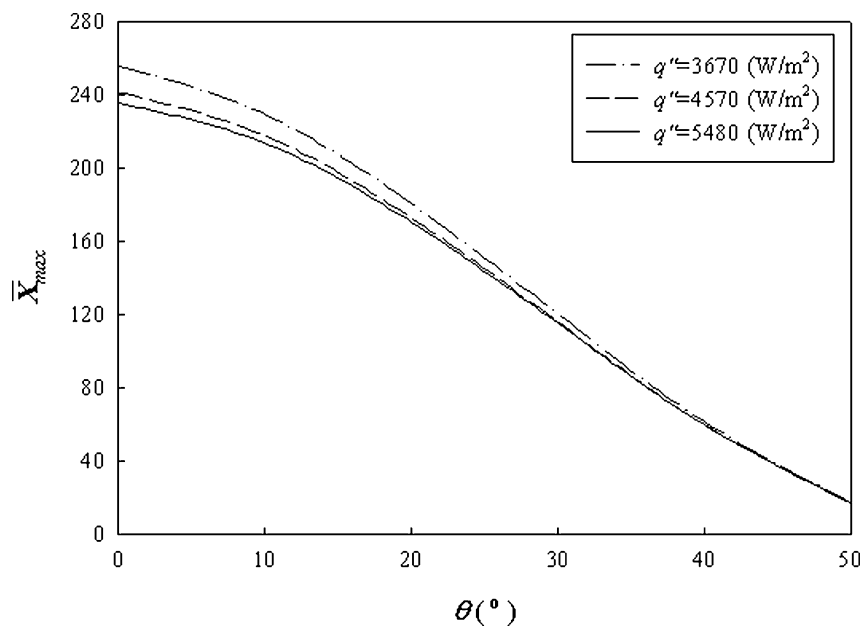

Fig. 12. Wetted axial length versus contact angle at different heat fluxes (ethanol and $\beta=15^{\circ}$ )

pipes. Figures 11 and 12 are the correction between heat flux and wetted axial length at different contact angles. The working fluids are methanol and ethanol respectively. The heat fluxes are 5150,6600 and $8070 \mathrm{~W} / \mathrm{m}^{2}$ for methanol, and $3670,4570,5480 \mathrm{~W} / \mathrm{m}^{2}$ for ethanol. The inclination angle is $15^{\circ}$, and the contact angles range from $0.1^{\circ}$ to $50^{\circ}$. Both figures show that the wetted axial length has maximum value when the contact angle is $0.1^{\circ}$, and the value decreases with the increase of the contact angle. The slope of the curve is steep, which evidences that the influence of contact angle on the capillary performance is enormous. Therefore, the surface modification is a very important technique in improving the capillary performance of microgrooves.

Figures 13 and 14 are the correction between the hydraulic diameter and the wetted axial length. The working fluids are methanol and ethanol, heat fluxes and inclined angle, $5150 \mathrm{~W} / \mathrm{m}^{2}$ and $3670 \mathrm{~W} / \mathrm{m}^{2}$, and $15^{\circ}$ respectively. In order to interpret the influence of the hydraulic diameter on the capillary performance, the angle of the bottom of the triangular microgroove is varied from 


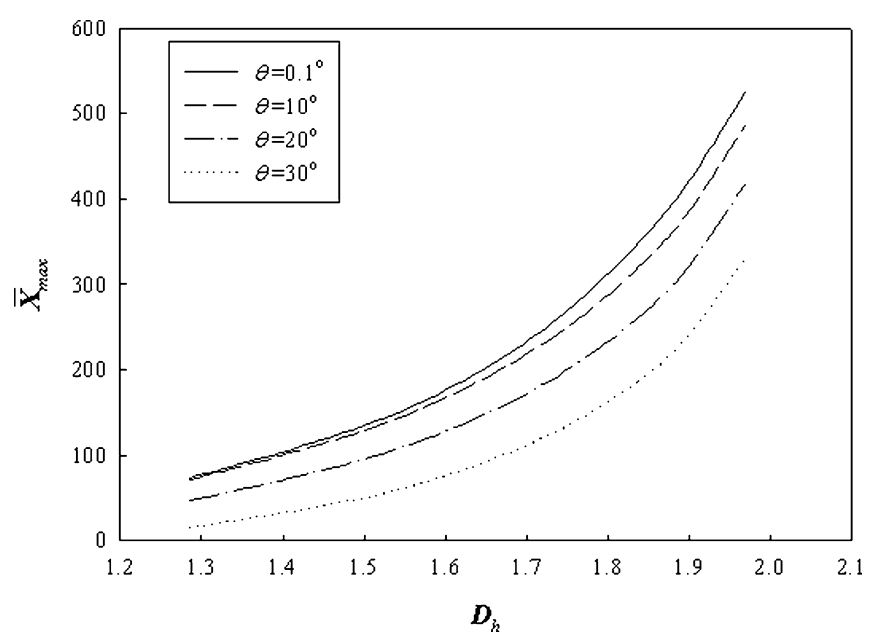

Fig. 13. Wetted axial length versus hydraulic diameter at different contact angles (methanol and $q^{\prime \prime}=5150 \mathrm{~W} / \mathrm{m}^{2}$ )

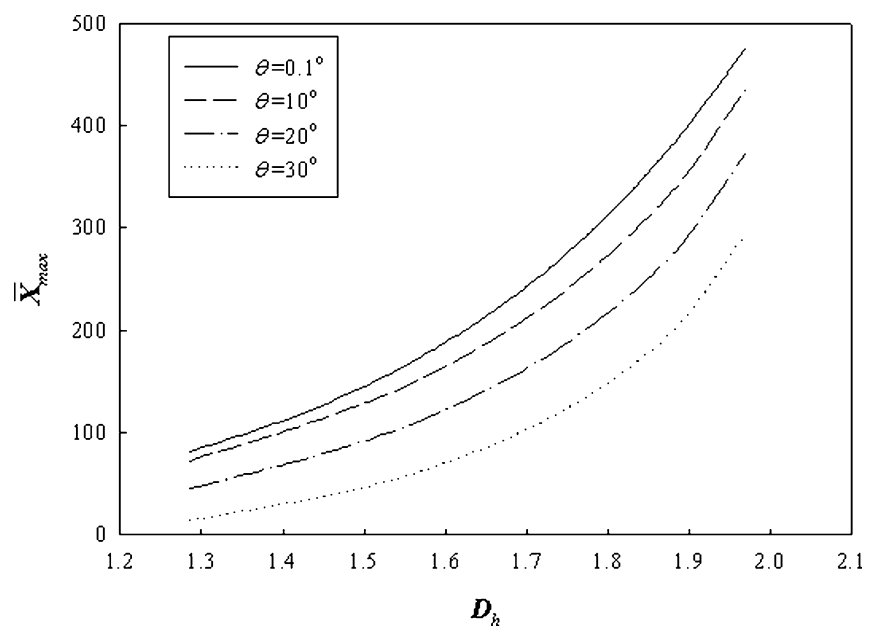

Fig. 14. Wetted axial length versus hydraulic diameter at different contact angles (ethanol and $q^{\prime \prime}=5150 \mathrm{~W} / \mathrm{m}^{2}$ )

$10^{\circ}$ to $50^{\circ}$. Thus, the hydraulic diameter $D_{h}$ ranges 1.33 to $1.95\left(D_{h}=d_{h} / w, d_{h}=257.1-393.9 \mu \mathrm{m}\right)$. The width of the groove $(2 w)$ is constant to maintain the value of capillary force. The results show that the wetted axial length increases with the increase of the hydraulic diameter. The comparison between the maximum and minimum hydraulic diameter shows that the hydraulic diameter increases merely 1.5 times but the wetted axial length increases about 5.8 times. It is believed that the primary reason is the increase of the flow cross-section area.

\section{Conclusion}

The theoretical analysis is established based on a onedimensional contact angle including differential equations and algebraic equations. The predicted values are compared with experimental data. The algebraic solutions are very close to the experimental data, and the numerical solutions are also presented as the qualitative study for the experiment. By numerically solving the governing equation, the wetted axial length, curvature radius, cross-section area of liquid surface, and pressure and velocity distribution along axial direction are obtained. Meanwhile, the mutual effects among momentum, body force, capillary force, and friction force of the liquid-vapor interface are also solved. The results of the algebraic prediction show that the wetted axial length decreases with contact angle, but increases with the decrease of the hydraulic diameter. The theoretical analysis presented here can be efficiently used in the wick design of micro heat pipes to achieve better capillary performance.

\section{References}

1. Holm FW; Goplen SP (1979) Heat transfer in the meniscus thin-film transition region. ASME J Heat Transfer 101: 543547

2. Xu X; Carey VP (1990) Film evaporation from a microgrooved surface - an approximate heat transfer model and its comparison with experimental data. J Thermophys 4: 512-520

3. Stephan PC; Busse CA (1992) Analysis of the heat transfer coefficient of grooved heat pipe evaporator walls. Int J Heat Mass Transfer 35: 383-391

4. Stroes G; Rohloff T; Catton I (1992) An experimental study of the capillary forces in rectangular channels vs. triangular channels. 1992 ASME National Heat Transfer Conference, ASME HTD 200, pp. 1-8

5. Ha JM; Peterson GP (1994) Analytical prediction of the axial dryout point for evaporating liquids in triangular microgrooves. ASME J Heat Transfer 116: 498-503

6. Khrustalev D; Faghri A (1995) Heat transfer during evaporation on capillary-grooved structures of heat pipes. ASME J Heat Transfer 117: 740-747

7. Ha JM; Peterson GP (1998) Capillary performance of evaporating flow in micro grooves: an analytical approach for very small tilt angles. ASME J Heat Transfer 120: 452-457

8. Peterson GP; Ha JM (1998) Capillary performance of evaporating flow in micro grooves: an approximate analytical approach and experimental investigation. ASME J Heat Transfer 120: 743-751

9. Sheu TS; Ding PP; Lo IM; Chen PH (2000) Effect of surface characteristics on capillary flow in triangular microgrooves. Exp Thermal Fluid Sci 22: 103-110

10. Ayyaswamy PS; Catton I; Edwards DK (1974) Capillary flow in triangular grooves. J Appl Mech 41: 332-336 